\title{
Sealing a large double lobe left atrial appendage with two Occlutech occluders
}

\author{
Barbara Bellmann ${ }^{1 *}$, Caroline Kleinecke ${ }^{2 *}$, Johannes Brachmann², Jai-Wun Park ${ }^{1,2}$ \\ ${ }^{1}$ Department of Cardiology, Campus Benjamin Franklin, \\ Charité - Universitätsmedizin Berlin, Germany \\ ${ }^{2}$ Department of Cardiology and Intensive Care Medicine, \\ Klinikum Coburg, Germany
}

This article reports the case of a 63-year-old male who was admitted for left atrial appendage (LAA) closure (LAAC) due to permanent atrial fibrillation with indication for oral anticoagulation $\left(\mathrm{CH}_{2} \mathrm{ADS}_{2}\right.$-VASc score 3$)$ and high bleeding risk (HAS-BLED score 3). Transesophageal echocardiography (TEE) was performed to rule out LAA thrombi, to determine LAA-size and to guide the procedure. After femoral venous access and transseptal puncture an LAA-angiography was performed. Both TEE and angiography revealed a large double lobe LAA with a common ostium of $30 \mathrm{~mm}$. Both lobes had a landing zone of $14 \mathrm{~mm}$ and a length of $35 \mathrm{~mm}$. It would have been desirable to close the LAA with one device. This was not possible because of the cactus morphology with two early outgoing lobes. Other devices would probably have left significant leakage or would have displaced each other due to a significantly greater radial force. Two Occlutech LAA occluders with a size of $18 \mathrm{~mm}$ were chosen, because of their lower radial force. The Occlutech LAA-occluder (Occlutech GmbH, Jena, Germany, Fig. 1A) consists of a self-expanding, flexible nitinol mesh with a tapered cylindrical shape; the proximal part has a larger diameter to seal the LAA-orifice; the loops at the distal rim aid to keep the implanted device in position; the surface of the occluder is covered with a non-woven, bio-stable Poly (carbonate) urethane layer. Both devices were successfully implanted in the upper and lower lobe (Fig. 1B-E). TEE after 3 months revealed complete closure of the LAA (Fig. 1F).

Conflict of interest: Dr. Park is a consultant for Occlutech GmbH, Jena, Germany

Address for correspondence: Caroline Kleinecke, MD, Department of Cardiology and Intensive Care Medicine, Klinikum Coburg, 96450 Coburg, Germany, tel: + 49 17670806948, fax +49 9561 226349, e-mail: carolinekleinecke@web.de

Received: 20.01.2017 Accepted: 29.09.2017

*The first two authors contributed equally to this work. 


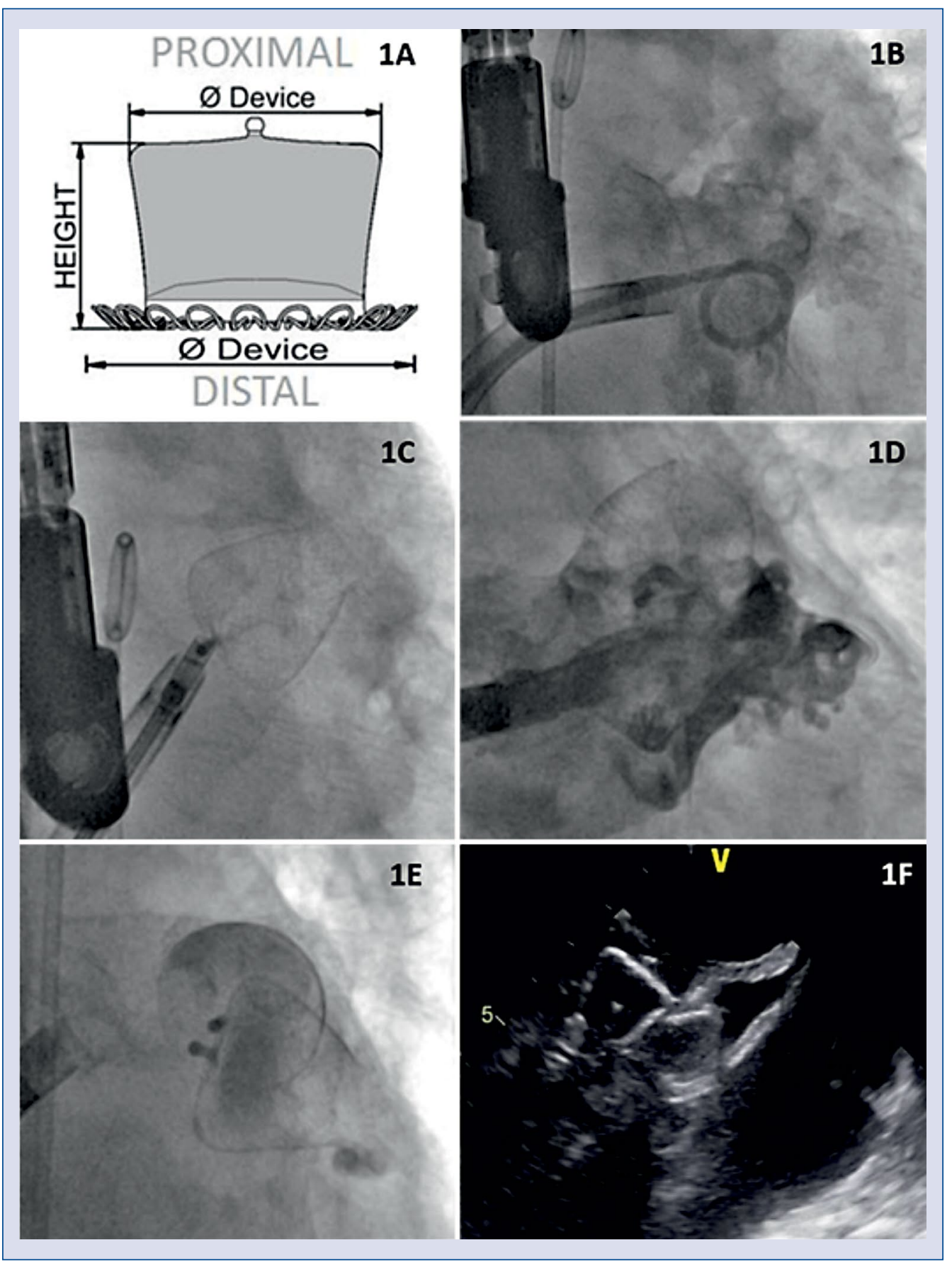

Figure 1. A. Schematic drawing of the Occlutech left atrial appendage occluder (provided by Occlutech $\mathrm{GmbH}$, Jena, Germany). The device's proximal section has a larger diameter to seal the orifice and a distal loop rim that helps to maintain the position of the implanted device; B-E. Step-by-step illustration of the procedure. Left atrial appendage angiography showed a large double lobe left atrial appendage (B). Deployment of the first occluder (C). Angiography of the remaining lobe (D). Deployment of the second occluder (E); F. Transesophageal echocardiography showing complete closure of the left atrial appendage. 